\title{
Tratamento cirúrgico para ectasia ânulo-aórtica
}

\author{
Ricardo GARCÍA-MACEDO*, Renato A. K. KALIL*, Paulo Roberto PRATES*, Fernando A. LUCCHESE, \\ João Ricardo SANT'ANA*, Edemar M. PEREIRA*, Altamiro Reis COSTA*, Raul Feck A. LARA, Ivo A. \\ NESRALLA*, Porto Alegre, RS.
}

GARCIA-MACEDO, R.; KALIL, R. A. K.; PRATES, P.R.; LUCCHESE, F.A.; SANT'ANA, J.R.; PEREIRA, E. M.; COSTA, A.R.; LARA, R. F. A.; NESRALLA, I. A. - Tratamento cirúrgico para ectasia ânulo-aórtica. Rev. Bras. Cir. Cardiovasc., 1 (1): 44-48, 1986.

RESUMO: Foram estudados 27 pacientes submetidos a implante de tubo de Dacron valvulado (segundo técnica de Bentall-De Bono), entre 1976 e 1985. Todos apresentavam ectasia ânulo-aórtica, que era devida a necrose cística da média em 23 pacientes $(85,2 \%)$, dissecção crônica de aorta em 3 pacientes $(11,1 \%)$ e aortite luética em 1 paciente $(3,7 \%)$. A faixa etária foi de 29 a 64 anos $(m=48$ anos). Três eram do sexo feminino e 24, do masculino. Foi utilizado tubo de Dacron 28 ou $30 \mathrm{~mm}$ em todos. As válvulas utilizadas foram: de dura-máter em 7 pacientes, de aorta heteróloga em 9 e metálica em 11 pacientes. A classe funcional era I-II em 7 pacientes e III-IV em 19 pacientes (NYHA). Houve 1 óbito $(3,7 \%$ ) pós-operatório imediato, por sangramento incontrolável e insuficiência renal aguda. As complicaçōes mais freqūentes foram arritmias (13 pacientes, $48 \%$ ) e hemorragia ( 3 pacientes, $11 \%$ ). No pós-operatório tardio faleceram 9 pacientes, pelas seguintes causas: morte súbita ( 2 pacientes, $7,4 \%$ ), insuficiência cardiaca aguda ( 2 pacientes, $7,4 \%$ ), hemorragia após reoperação (1 paciente, $3,7 \%$ ), infecção hospitalar ( 1 paciente, $3,7 \%$ ), endocardite infecciosa (1 paciente, $3,7 \%$ ) e causa acidental $(7,4 \%)$. Cinco pacientes $(18,5 \%)$ foram reoperados no pós-operatório tardio, 2 por disfunçâo de prótese, 2 por endocardite infecciosa sobre tubo de Dacron e 1 por fistula perivalvular. A curva atuarial mostrou uma probabilidade de sobrevida de $83,7 \%$ até o $3^{\circ}$ ano, $61 \%$ aos 5 anos e $42,7 \%$ do $6^{\circ}$ ao $9^{\circ}$ ano. No fim do período, os 17 pacientes sobreviventes encontram-se em classe funcional I-II $(p<0,001)$, em relação ao pré-operatório. Concluímos que a cirurgia de Bentall \& De Bono, para ectasia ânulo-aórtica, está associada a uma mortalidade operatória baixa. Houve melhora significativa na capacidade funcional dos pacientes, após o tratamento, e a sobrevida a longo prazo foi satisfatória.

DESCRITORES: ectasia ânulo-aórtica, cirurgia.

\section{INTRODUÇÃO}

Em 1961, ELLIS et alii ${ }^{5}$ propuseram o termo ectasia ânulo-aórtica para os aneurismas de aorta ascendente com dilatação dos seios de Valsalva e do anel aórtico, com perda de coaptação dos folhetos valvares e regurgitação aórtica. Na maioria dos casos, está associada a alterações degene- rativas da parede aórtica do tipo necrose cística da média.

O termo não foi reservado somente para os casos de médio-necrose, se não também para outras etiologias menos freqüentes, como a aterosclerose, aortite luética, dissecção crônica de aorta e outras. 
Esta patologia foi, e continua sendo, considerada um desafio para os cirurgiōes, não só pelas dificuldades técnicas, como pela alta mortalidade e complicações pós-operatórias.

Em 1968, BENTALL \& DE BONO ${ }^{1}$ descreveram a técnica de substituição radical da aorta ascendente com tubo de Dacron valvulado, procedimento que melhorou significativamente a expectativa de vida desses pacientes.

É nosso objetivo apresentar, neste trabalho, nossa experiência com esta técnica cirúrgica em nove anos de uso.

\section{MATERIAL E MÉTODO}

Foram estudados 27 pacientes submetidos a implante de tubo aórtico valvulado, no período de janeiro de 1976 a maio de 1985 , no Instituto de Cardiologia do Rio Grande do Sul.

\section{TÉCNICA CIRÚRGICA}

A circulação extracorpórea foi estabelecida por canulação de átrio direito e artéria femoral, com drenagem de átrio esquerdo, sob hipotermia moderada a $28-30^{\circ} \mathrm{C}$, em todos os casos. A aorta foi pinçada distalmente à zona aneurismática e realizada aortotomia longitudinal para exame. Foi infundida solução cardioplégica diretamente nos óstios coronários, bem como mantida hipotermia tópica com solução salina gelada. Nos primeiros casos da série, foi utilizada apenas hipotermia tópica. O tubo de Dacron-Woven a ser implantado foi preparado no momento da cirurgia, suturando-se a prótese escolhida a uma de suas extremidades. Os foIhetos aórticos, geralmente amplos, de consistência delicada, foram sempre ressecados. $O$ tubo foi implantado com pontos isolados de poliéster 2-0, de forma semelhante a uma prótese aórtica comum. Após sua fixação, foram feitos os orifícios laterais para implante dos óstios coronários, o que foi realizado com suturas contínuas de prolene 4-0, às vezes ancoradas em feltros de Teflon. A sutura distal do tubo à porção sadia da aorta foi realizada de forma contínua com prolene 4-0. Em alguns casos, foi cerclada a aorta distal com tecido ou tubo de Dacron. Após completadas todas as suturas, a pinça aórtica foi aberta para pesquisa e controle de pontos sangrantes. A parede aórtica foi, então, ajustada e suturada por sobre o tubo implantado. O paciente foi reaquecido, os batimentos cardiacos, recuperados e encerrada a perfusão extracorpórea.

Vinte e quatro pacientes eram do sexo masculino $(88,9 \%)$ e três do sexo feminino $(11,1 \%)$, com idades entre 29 e 64 anos $(m=48$, DP $= \pm$ 9 anos). Nos 27 pacientes, foi feito diagnóstico clínico, angiográfico e intraoperatório de ectasia ânuloaórtica.

Três pacientes $(11,1 \%)$ apresentavam dissecção crônica de aorta tipo II de DE BAKEY ${ }^{4}, 1$ (3,7\%) aortite luética e $23(85,2 \%)$ necrose cística da média confirmada por estudo anátomo-patológico. Destes últimos, 3 pacientes apresentavam os clássicos estigmas da síndrome de Marfan.

Dos 27 pacientes, $7(25,9 \%)$ se apresentavam em classe funcional I-II (critério NYHA) e $20(74,1 \%)$ em classe funcional III-IV.

Todos foram submetidos a implante de tubo de Dacron valvulado, segundo a técnica descrita por BENTALL \& DE BONO ${ }^{1}$, com pequenas modificaçōes.

As próteses utilizadas foram dura-máter em 7 pacientes $(25,9 \%)$, de aorta heteróloga em 9 $(33,3 \%)$, metálicas de bola em $4(4,8 \%)$ e metálicas de disco em 7 (25,9\%).

O seguimento dos pacientes foi de 1 mês a 9 anos ( $m=3$ anos, DP $=2,5$ anos) com controles clínico-radiológicos periódicos e angiográficos em alguns casos.

Os métodos estatísticos utilizados foram o teste de McNemar para análise de variância das classes funcionais, pré e pós-operatória (considerando-se como significativos os valores $p<0,05$ ) e a curva atuarial para estimativa de sobrevida.

\section{RESULTADOS}

\section{Imediatos}

Houve 1 óbito $(3,7 \%)$ pós-operatório imediato em um paciente de 40 anos, portador de médio-necrose cística, que se apresentou com aneurisma de aorta ascendente e insuficiência aórtica recidivante. Este paciente já havia sido submetido, 1 ano antes, a aortoplastia e implante de prótese valvular. A causa do óbito foi sangramento incontrolável, baixo débito cardíaco e insuficiência renal aguda.

Ocorreram complicações pós-operatórias imediatas em $51,9 \%$ dos pacientes, sendo as mais freqüentes as arritmias $(48,1 \%)$ e, destas, as ventriculares, assim como o sangramento $(11,1 \%)$, que motivou reexploração cirúrgica em 3 casos, nas primeiras $24-48$ horas. Outras complicaçōes menos freqüentes foram o edema cerebral, insuficiência cardíaca e insuficiência renal aguda (Tabela 1). 
TABELA 1

Complicaçōes pós-operatórias imediatas * n: de casos com complicaçōes: $14-51,85 \%$

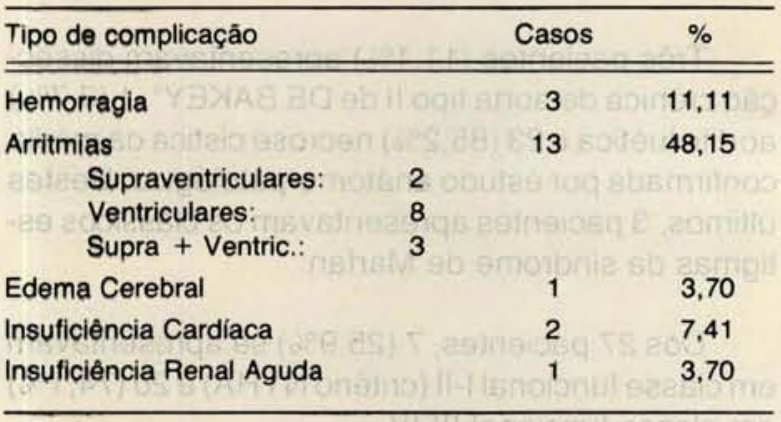

- Alguns pacientes apresentaram mais de uma complicação.

\section{Tardios}

Nove pacientes faleceram no pós-operatório tardio, $2(7,4 \%)$ por morte súbita e $2(7,4 \%)$ por insuficiência cardiaca aguda, $1(3,7 \%)$ por endoi cardite infecciosa, $1(3,7 \%)$ por sangramento incon- taram substituição de válvula de dura-máter por metálica aos 9 e 30 meses, $2(7,4 \%)$ por endocardite infecciosa e que fizeram troca de tubo de Dacron aos 5 e 9 meses, sendo que o último faleceu no pós-operatório imediato, e 1 (3,7\%) por pseudo-aneurisma com insuficiência aórtica, em que foi ressuturado o óstio coronário esquerdo.

\section{TABELA 2}

Mortalidade e causas de obito

\begin{tabular}{lll}
\hline Causas & No $(\%)$ & $\begin{array}{c}\text { No } \\
\text { total }\end{array}$ \\
\hline \hline
\end{tabular}

Pós-operatório imediato

Pós-operatório tardio Morte súbita

Insuficiência cardíaca

aguda

Endocardite infecciosa

Sangramento pós re-opera-

tório

Infecçāo hospitalar

Acidental

3,70

$9 \quad 33,33$

$2(7,41)$

$2(7,41)$

$1(3,70)$

$1(3,70)$

$1(3,70)$

$2(7,41)$

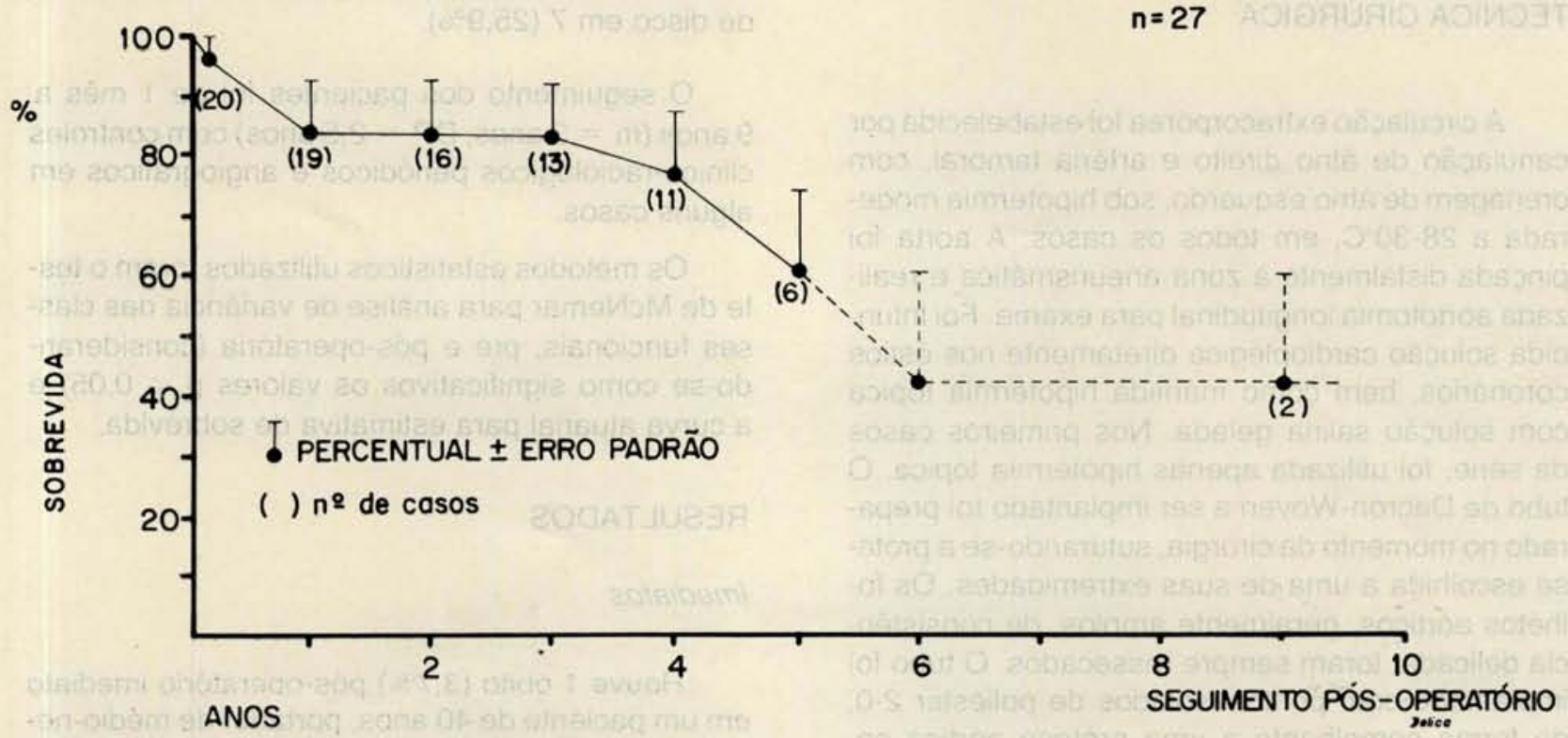

Fig. 1 - Curva atuarial de sobrevida a longo prazo.

trolável no pós-operatório de reoperação, 1 (3,7\%) por infecção intra-hospitalar e $2(7,4 \%)$ por causa acidental (Tabela 2). A probabilidade de sobrevida estimada pelo método atuarial (Figura 1) foi de $83,7 \%$ aos 3 anos, $61 \%$ aos 5 anos e $42,7 \%$ dos 5 aos 9 anos.

As complicações tardias foram: disfunção de prótese em 3 pacientes $(11,1 \%)$, endocardite infecciosa em $3(11,1 \%)$ e acidente vascular encefálico de possível causa embólica em 2 (7,4\%) (Tabela 3). Cinco pacientes $(18,5 \%)$ foram reoperados, 2 $(7,4 \%)$ por disfunção de prótese e que necessi-
TABELA 3

Complicaçōes pós-operatórias tardias

\begin{tabular}{lcr}
\hline \hline Tipo de complicação & Casos & $\%$ \\
\hline Disfunção de prótese & 3 & 11,11 \\
Endocardite infecciosa & 3 & 11,11 \\
Acidente vascular cerebral & 2 & 7,41 \\
\hline T O T A L & 8 & 29,63 \\
\hline
\end{tabular}

Dos 17 pacientes sobreviventes em junho de 1985, todos achavam-se em classe funcional I-II, 
melhoria estatisticamente significativa em relação ao pré-operatório (Teste de McNemar $-X^{2} 12,00$ $-p<0,001)$.

\section{COMENTÁRIOS}

Antes de haver a disponibilidade de enxertos vasculares e próteses valvulares, o tratamento dos aneurismas de aorta ascendente se limitava ao emprego de aneurismorrafia e valvoplastia.

Em 1956, COOLEY \& DE BAKEY² descreveram a substituição aórtica supracoronária com enxerto sintético. Posteriormente, outros autores relataram a associação desta técnica com valvoplastias ou implante de prótese valvular.

Em 1964, WHEAT et alii ${ }^{10}$ descrevem a ressecção radical da parede aórtica, conservando só as pequenas zonas de tecido adjacente aos óstios coronários e implante de prótese aórtica, separadamente.

Estas técnicas não eliminavam o potencial desenvolvimento de dilatação dos seios de Valsalva e era importante o sangramento das linhas de sutura e através do Dacron, assim como a formação de fístulas periprotéticas e aneurismas recidivantes.

A técnica de reimplante total, com tubo valvulado descrita por BENTALL \& DE BONO', trouxe vantagens significativas, como a diminuição do tempo operatório, exclusão total da área afetada e diminuição do sangramento pós-operatório.

A técnica por nós utilizada não difere muito da descrita por estes autores. Em nossa série, não tivemos nenhum caso de dissecção dos óstios coronários e, em todos os pacientes, foi possível a sutura periostial direta ao tubo de Dacron, às vezes com pontos ancorados com feltros de Teflon. Não foi necessária a interposição de enxerto de safena.

A variedade de próteses utilizadas corresponde às mudanças de critério de prótese ideal e aos resultados da experiência cirúrgica acumulada nos últimos 15 anos. Atualmente, preferimos o uso de válvulas metálicas de disco com fluxo central. Porém, dos 6 primeiros pacientes, descritos ${ }^{6}$ em estudo prévio e que implantaram válvulas de dura-máter, os dois primeiros são os que maior sobrevida apresentam (9 anos), sem complicaçōes atribuídas à prótese.

A mortalidade hospitalar foi baixa $(3,7 \%)$ e a sobrevida calculada pelo método atuarial foi boa, com resultados superponíveis aos relatados na literatura $^{8.9}$

O índice de reoperações foi de $18,5 \%$ ( 5 casos), percentual elevado, comparado ao de outros autores ${ }^{7,8}$, porém duas destas reoperaçōes foram motivadas por disfunção de válvula de dura-máter, que, atualmente, não estamos utilizando.

A mortalidade hospitalar de reoperação foi de $20 \%$.

S. CRAWFORD ${ }^{3}$, em estudo recente sobre reoperações em pacientes com doença aneurismática recorrente de aorta torácica, faz referência a 34 casos reoperados, em pacientes portadores de doença degenerativa da camada arterial média. Os autores salientam o aspecto profilático do emprego de técnicas radicais nesta doença. Assim, a técnica mais utilizada nas reoperações foi o implante de tubo valvulado. Têm sido descritas ${ }^{9}, \operatorname{como}$ complicaçōes pós-operatórias tardias, a compressão do tubo e a estenose de artéria coronária, complicaçōes estas que, até o final deste estudo, não temos observado.

Por último, concluímos que, em nossa série, a etiologia mais freqüente de ectasia ânulo-aórtica foi a necrose cística da média. Após o tratamento cirúrgico, ocorreu melhora significativa na capacidade de funcional dos pacientes.

Pensamos que a técnica de BENTALL \& DE BONO' é o procedimento de escolha para aqueles casos de aneurisma de aorta ascendente com insuficiência aórtica, sobretudo os devidos a doença degenerativa da aorta. Os procedimentos mais convencionais não excluem radicalmente os tecidos patológicos, mantendo a perspectiva de uma reoperação com indice de mortalidade bem maior. 
GARCÍA-MACEDO, R.; KALIL, R. A. K.; PRATES, P. R.; LUCCHESE, F. A.; SANTANA, J. R.; PEREIRA, E. M.; COSTA, A. R.; LARA, R. F. A.; NESRALLA, I. A. - Surgical treatment for annulo-aortic ectasia - Rev. Bras. Cir. Cardiovasc., 1(1): 44-48, 1986.

ABSTRACT: This is a report of 27 patients presenting annulo-aortic ectasia submitted to surgical correction with a composite valve-tube graft (Bentall-De Bono technique), between 1976 and 1985 . Twenty three (85.2\%) had cystic medial necrosis, $3(11,1 \%)$ were chronic aortic disections and $1(3.7 \%)$ was luetic aortitis. Age ranged from 29 to 64 years $(m=48)$. Three were female and 24 male patients. The valves used were homologous dura máter in 7 cases, heterologous aorta in 9 and mechanical prosthesis in 11. Functional class (NYHA) was I-II in 7 and III-IV in 9 patients. There was $1(3.7 \%)$ hospital death, due to bleeding and renal failure. Significant early complications were represented by arrhythmias in 13 cases (48\%) and bleeding in $3(11 \%)$. In the late follow-up there were 9 deaths, due to: sudden death (2 patients, $7.4 \%$ ), bleeding in reoperation (1 patient, 3.7\%), hospital infection (1 patient, $3.7 \%$ ), infectious endocarditis (1 patient, $3.7 \%$ ) and accidental causes ( 2 patients, $7.4 \%$ ). Five patients $(18.5 \%)$ needed reoperation in the late follow-up: 2 due to bioprosthetic failure, 2 for replacement of an infected Dacron tube and 1 for repair of pseudo-aneurysm and periprosthetic leak. The actuarial survival curve showed a probability of $83.7 \%$ up to the 3 rd. year, $61 \%$ for the 5 th. year and $42.7 \%$ from the 6 th. to the 9 th. year of follow-up. The 17 patients surviving are in functional class I-II (NYHA). The composite valve-Dacron tube graft (Bentall-De Bono) technique for correction of annulo-aortic ectasia is associated with a low hospital mortality. The improvement in functional class is significant. There have been a small number of complications and the long term survival is very satisfactory.

DESCRIPTORS: annulo-aortic ectasia, surgery.

\section{REFERÊNCIAS BIBLIOGRÁFICAS}

1 BENTALL, H. \& DE BONO, A. - A technique for complete replacement of the ascending aorta. Thorax, 23 : 338-339, 1968.

2 COOLEY, D. A. \& DE BAKEY, M. E. - Resection of entire ascending aorta in fusiform aneurysm using cardiac bypass. JAMA , 162: 1158-1159, 1956.

CRAWFORD, E.S.; CRAWFORD, J.L.; SAFI, H. J.; COSELLI, J. S. - Redo operations for recurrent aneurysmal disease of the ascending aorta and transverse aortic arch. Ann. Thorac. Surg., 40: 439-455, 1985.

4 DE BAKEY, M. E.; HENLY, W. S.; COOLEY, D. A.; MORRIS Jr., G. C.; CRAWFORD, E. S., BEALL, A.C. Surgical management of dissecting aneurysms of the aorta. J. Thorac. Cardiovasc. Surg., 49: 130-149, 1965.

5 ELLIS, P.R.; COOLEY, D. A.; DE BAKEY, M. E. - Clinical considerations and surgical treatment of annulo-aortic ectasia. J. Thorac. Cardiovasc. Surg., 42: 363-370, 1961.
6 KALIL, R. K.; AZAMBUJA, P. C,; BERTOLETTI, V.E.; LUCCHESE, F. A.; PRATES, P. R.; NESRALLA, I. A. Surgical treatment of annuloaortic ectasia with composite grafts including homologous dura mater valves. Ann. Thorac. Surg., 26: 142-148, 1978.

7 KOUCHOUKOS, N. T.; KARP, R. B.; LELL, W. A. - Replacement of the ascending aorta and aortic valve with a composite graft: results in 25 patients. Ann. Thorac. Surg., 24: 140-148, 1977.

8 MAYER Jr., J. E.; LINDSAY, W. G.; WANG, Y.; JORGENSEN, C. R.; NICOLOFF, D. M. - Composite replacement of the aortic valve and ascending aorta. J. Thorac. Cardiovasc. Surg., 76: 816-823, 1978.

9 MORENO-CABRAL, C. E.; MILLER, D. C.; MITCHELL, R. S.; STINSON, E. B.; OYER, P.E.; JAMIESON, S. W.; SHUMWAY, N. E. - Degenerative and atherosclerotic aneurysms of the thoracic aorta. $J$. Thorac. Cardiovasc. Surg., 88: 1020-1032, 1984.

10 WHEAंT, M. W.; WILSON, J. R.; BARTLEY, T. D. - SUCcessful replacement of the entire ascending aorta and aortic valve. JAMA, 188: 717-719, 1964. 